\title{
Transmit Power Allocation for a Modified V-BLAST System
}

\author{
Seung Hoon Nam, Oh-Soon Shin, and Kwang Bok (Ed) Lee, Member, IEEE
}

\begin{abstract}
In this letter, we present a modification of Vertical Bell Laboratories Layered Space-Time (V-BLAST), and propose an effective transmit power allocation (TPA) scheme for the modified system. The proposed TPA scheme minimizes the uncoded biterror rate (BER) averaged over all detection stages, and requires small feedback overhead. Simulation results show that the modified V-BLAST system with the proposed TPA scheme provides a significant reduction in the uncoded BER compared with the conventional V-BLAST system. When the minimum mean-square error nulling is adopted, the modified V-BLAST system is found to achieve the uncoded BER performance comparable to that of the maximum-likelihood detection for the conventional V-BLAST architecture.
\end{abstract}

Index Terms-Bit-error rate (BER), detection ordering, multiple-input multiple-output (MIMO) system, transmit power allocation (TPA), Vertical Bell Laboratories Layered Space-Time (V-BLAST).

\section{INTRODUCTION}

$\mathbf{M}$ ULTIPLE-INPUT multiple-output (MIMO) systems can provide enormous capacities through appropriate space-time processing [1]-[3]. A spatio-temporal processing architecture studied in [3] is a means to approach the theoretical capacity limit of MIMO systems. This system requires a feedback mechanism and complex processing at both the transmitter and receiver. On the other hand, Diagonal Bell Laboratories Layered Space-Time (D-BLAST) in [4] eliminates the need for feedback. However, this system requires a complex coding structure that makes the detection procedure complicated. In [5], Vertical BLAST (V-BLAST) has been proposed as a simplified version of D-BLAST. This system does not need a complex coding structure, unlike a D-BLAST. Simple coding and detection structures make the V-BLAST attractive.

There have been some attempts to improve the original V-BLAST system [6]-[10]. One such approach is to incorporate an effective transmit power allocation (TPA) into the original V-BLAST, where equal power is assigned to transmit antennas [5]. Transmit power is adapted according to the

Paper approved by A. Lozano, the Editor for Wireless Communication of the IEEE Communications Society. Manuscript received March 1, 2003; revised May 27, 2003; October 13, 2003; and November 7, 2003. This work was supported in part by the Brain Korea 21 Project. This paper was presented in part at the 13th IEEE Symposium on Personal, Indoor and Mobile Radio Communications, Lisbon, Portugal, September 2002.

S. H. Nam was with the School of Electrical Engineering and Computer Science, Seoul National University, Seoul 151-742, Korea. He is now with Samsung Advanced Institute of Technology, Suwon 440-600, Korea (e-mail: seunghoon.nam@samsung.com).

O.-S. Shin was with the School of Electrical Engineering and Computer Science, Seoul National University, Seoul 151-742, Korea. He is now with the Division of Engineering and Applied Sciences, Harvard University, Cambridge, MA 02138 USA (e-mail: osshin@ fas.harvard.edu).

K. B. Lee is with the School of Electrical Engineering and Computer Science, Seoul National University, Seoul 151-742, Korea (e-mail: klee@ snu.ac.kr).

Digital Object Identifier 10.1109/TCOMM.2004.831368 channel condition, which necessitates a feedback mechanism. However, a feedback overhead needed for TPA is relatively small, compared with the system in [3]. In [6]-[8], TPA schemes with small feedback overhead have been investigated to increase the capacity of V-BLAST systems through rate adaptation [10]. However, there have been few attempts to improve the error-rate performance of MIMO systems for a given fixed rate.

In this letter, we present a modification of V-BLAST that incorporates TPA, and propose an effective TPA scheme to improve the bit-error rate (BER) performance. In the V-BLAST detection algorithm, a diversity order for an earlier detection stage is less than that for a later one [2], [5], [9]. Considering this characteristic and the effects of error propagation [9], it is apparent that early detection stages limit the overall performance. Although a detection-ordering scheme in [5] mitigates this problem, it may not sufficiently compensate for low diversity orders of early detection stages [9]. Hence, TPA for the modified V-BLAST may offer further improvement of early stages, enhancing the overall performance. We develop a TPA scheme that minimizes the BER. The transmit power determined at the receiver is transferred to the transmitter. The feedback overhead for the proposed TPA is relatively small, compared with the system in [3], since the feedback information contains only the transmit power rather than full channel state information.

\section{MODIFIED V-BLAST SYSTEM}

\section{A. System Description}

The modified V-BLAST system is equipped with $M$ transmit and $N(\geq M)$ receive antennas. In the transmitter, a data stream is demultiplexed into $M$ independent substreams, and then each substream is encoded into transmit symbols using the same modulation scheme. Based on the feedback information, the transmit power $P_{i}$ is assigned to the data symbol $x_{i}$, and the symbol is transmitted through the $i$ th transmit antenna. The receiver estimates the transmit symbols from the received signals at $N$ receive antennas, and determines the transmit power $P_{i}(i=1,2, \ldots, M)$.

The baseband equivalent of the $N$-dimensional received signal vector $\mathbf{y}=\left[\begin{array}{llll}y_{1} & y_{2} & \cdots & y_{N}\end{array}\right]^{T}$ at sampling instants may be expressed as

$$
\mathbf{y}=\mathbf{H P x}+\mathbf{n}
$$

where $\mathbf{x}=\left[\begin{array}{llll}x_{1} & x_{2} & \cdots & x_{M}\end{array}\right]^{T}$ denotes the transmit symbol vector with each element having the unit average power, and $\mathbf{H}$ denotes the $N \times M$ channel matrix, whose element $h_{n m}$ at the $n$th row and $m$ th column is the channel gain from the $m$ th transmit antenna to the $n$th receive antenna, and they are assumed to be independent and identically distributed (i.i.d.) 
complex Gaussian random variables with zero mean and unit variance. The elements of the $N$-dimensional noise vector $\mathbf{n}=\left[\begin{array}{llll}n_{1} & n_{2} & \cdots & n_{N}\end{array}\right]^{T}$ are assumed to be i.i.d. complex Gaussian random variables with zero mean and variance of $\sigma_{n}^{2}$. It is assumed that the channel estimation at the receiver is perfect, and that the receiver determines the transmit power $P_{i}(i=1,2, \ldots, M)$ for $M$ transmit antennas with the total power constraint $\sum_{i=1}^{M} P_{i}=M$, and sends the power to the transmitter through an error-free feedback channel. A diagonal matrix $\mathbf{P}=\operatorname{diag}\left(\sqrt{P_{1}}, \sqrt{P_{2}}, \ldots, \sqrt{P_{M}}\right)$ in (1) represents the transmit power. For the conventional V-BLAST system without feedback, $P_{i}=1$ for all $i$, and thus, $\mathbf{P}$ is equal to the $M \times M$ identity matrix $\mathbf{I}_{M}$.

\section{B. Detection Algorithm for the Modified V-BLAST System}

For a conventional V-BLAST system, a detection algorithm has been presented in [5]. The algorithm uses the linear nulling and successive interference cancellation processes to estimate $M$ transmit symbols. This algorithm can easily be modified for the modified V-BLAST system.

1) Detection Algorithm for ZF Nulling: With a TPA matrix $\mathbf{P}$ being introduced, the detection algorithm in [5] can be modified as follows.

Initialization:

$$
\begin{aligned}
i & \leftarrow 1 \\
\mathbf{y}_{1} & =\mathbf{y} \\
\mathbf{G}_{1} & =(\mathbf{H P})^{+}=\mathbf{P}^{-1} \mathbf{H}^{+} \\
k_{1} & =\underset{j}{\arg \min }\left\|\left\langle\mathbf{H}^{+}\right\rangle_{j}\right\|^{2}
\end{aligned}
$$

\section{Recursion:}

$$
\begin{aligned}
\mathbf{w}_{k_{i}} & =\left\langle\mathbf{G}_{i}\right\rangle_{k_{i}} \\
z_{k_{i}} & =\mathbf{w}_{k_{i}} \mathbf{y}_{i} \\
\hat{x}_{k_{i}} & =D\left(z_{k_{i}}\right) \\
\mathbf{y}_{i+1} & =\mathbf{y}_{i}-\hat{x}_{k_{i}}[\mathbf{H P}]_{k_{i}}=\mathbf{y}_{i}-\sqrt{P_{k_{i}}} \hat{x}_{k_{i}}[\mathbf{H}]_{k_{i}} \\
\mathbf{G}_{i+1} & =\left([\mathbf{H P}]_{\bar{k}_{i}}\right)^{+} \\
k_{i+1} & =\underset{j \notin\left\{k_{1}, \ldots, k_{i}\right\}}{\arg \min }\left\|\left\langle\left([\mathbf{H}]_{\bar{k}_{i}}\right)^{+}\right\rangle_{j}\right\|^{2} \\
i & \leftarrow i+1
\end{aligned}
$$

where $(\cdot)^{+}$denotes the Moore-Penrose pseudoinverse, $\|\cdot\|$ is the norm of a vector, $\langle\cdot\rangle_{j}$ is the $j$ th row of a matrix, $[\cdot]_{j}$ is the $j$ th column of a matrix, and $[\cdot]_{\bar{k}_{i}}$ is a matrix formed by zeroing the $k_{1}, k_{2}, \ldots, k_{i}$ th columns of the argument matrix. $D(\cdot)$ is the slicing operator associated with a modulation scheme, and $\hat{x}_{k_{i}}$ is the estimated value of $x_{k_{i}}$. In (2a)-(2k), $k_{i}$ is the symbol index detected at the $i$ th stage, and thus the sequence $\left\{k_{1}, k_{2}, \ldots, k_{M}\right\}$ is the detection order of $M$ transmit symbols. Note that this detection order is determined based on the signal-to-interference-plus-noise ratio (SINR) of transmit symbols with $\mathbf{P}=\mathbf{I}_{M}$, since not $\mathbf{H P}$ but $\mathbf{H}$ is used in (2d) and $(2 \mathrm{j})$. The rationale for this is that the TPA, to be described in Section III, is determined under the assumption that the detection procedure follows the detection order for $\mathbf{P}=\mathbf{I}_{M}$.
The nulling vector in $(2 \mathrm{f})$ can be rewritten as

$$
\mathbf{w}_{k_{i}}=\left\langle\mathbf{G}_{i}\right\rangle_{k_{i}}=\frac{\left\langle\left([\mathbf{H}]_{\bar{k}_{i-1}}\right)^{+}\right\rangle_{k_{i}}}{\sqrt{P_{k_{i}}}}=\frac{\mathbf{v}_{k_{i}}}{\sqrt{P_{k_{i}}}}
$$

where the vector $\mathbf{v}_{k_{i}} \triangleq\left\langle\left([\mathbf{H}]_{\bar{k}_{i-1}}\right)^{+}\right\rangle_{k_{i}}$ corresponds to the nulling vector of the $i$ th detection stage, when $\mathbf{P}=\mathbf{I}_{M}$. We define the postdetection SINR, which determines the performance of each stage, as the SINR of the decision statistic $z_{k_{i}}$ in (2f). The interference component is zero for zero-forcing $(\mathrm{ZF})$, but nonzero for minimum mean-square error (MMSE). From (2f) and (3), the postdetection SINR $\rho_{k_{i}}$ for the $k_{i}$ th symbol can be calculated as

$$
\rho_{k_{i}}=\frac{1}{\sigma_{n}^{2}\left\|\mathbf{w}_{k_{i}}\right\|^{2}}=\frac{P_{k_{i}}}{\sigma_{n}^{2}\left\|\mathbf{v}_{k_{i}}\right\|^{2}} .
$$

$\rho_{k_{i}}$ is a random variable and is related to the weight vector $\mathbf{v}_{k_{i}}$ and transmit power $P_{k_{i}}$ for the $k_{i}$ th symbol. The average performance of the $i$ th detection stage is determined from the probability distribution of $\rho_{k_{i}}$. The effects of $\mathbf{v}_{k_{i}}$ on the distribution of $\rho_{k_{i}}$ may be characterized in terms of a diversity order [9]. It is known that $\mathbf{v}_{k_{i}}$ in (4) provides the distribution of $\rho_{k_{i}}$ with a diversity order of $N-M+i$ [2], [9]. This indicates that later detection stages achieve higher diversity orders, when error propagation is not considered. The effects of transmit power $P_{k_{i}}$ on $\rho_{k_{i}}$ and the average performance will be discussed in Section IV.

2) Detection Algorithm for MMSE Nulling: Unlike the ZF nulling that removes the interference components completely but results in noise enhancement, the MMSE nulling compromises interference suppression and noise enhancement, such that the mean-square error (MSE) between the transmit symbol and estimate of the receiver is minimized. In the case of MMSE, the nulling matrix $\mathbf{G}_{i}$ in (2c) and (2i) should be changed as

$$
\begin{aligned}
\mathbf{G}_{1} & =(\mathbf{H P})^{H}\left((\mathbf{H P})(\mathbf{H P})^{H}+\sigma_{n}^{2} \mathbf{I}_{N}\right)^{-1} \\
\mathbf{G}_{i+1} & =\left([\mathbf{H P}]_{\bar{k}_{i}}\right)^{H}\left(\left([\mathbf{H P}]_{\bar{k}_{i}}\right)\left([\mathbf{H P}]_{\bar{k}_{i}}\right)^{H}+\sigma_{n}^{2}\left[\mathbf{I}_{N}\right]_{\bar{k}_{i}}\right)^{-1}
\end{aligned}
$$

where $(\cdot)^{H}$ denotes the conjugate transpose. Furthermore, the detection order should be determined from the SINR for the MMSE nulling. Correspondingly, (2d) and (2j) are modified to

$$
\begin{aligned}
k_{1} & =\underset{j}{\arg \max } \frac{\left|\left(\mathbf{G}_{1}^{\prime} \mathbf{H}\right)_{j j}\right|^{2}}{\sigma_{n}^{2}\left\|\left\langle\mathbf{G}^{\prime}{ }_{1}\right\rangle_{j}\right\|^{2}+\sum_{\ell \neq j}\left|\left(\mathbf{G}^{\prime}{ }_{1} \mathbf{H}\right)_{j \ell}\right|^{2}} \\
k_{i+1} & =\underset{j \notin\left\{k_{1}, \ldots, k_{i}\right\}}{\arg \max } \frac{\left|\left(\mathbf{G}^{\prime}{ }_{i+1}[\mathbf{H}]_{\bar{k}_{i}}\right)_{j j}\right|^{2}}{\sigma_{n}^{2}\left\|\left\langle\mathbf{G}^{\prime}{ }_{i+1}\right\rangle_{j}\right\|^{2}+\sum_{\ell \neq j}\left|\left(\mathbf{G}^{\prime}{ }_{i+1}[\mathbf{H}]_{\bar{k}_{i}}\right)_{j \ell}\right|^{2}}
\end{aligned}
$$

where $\mathbf{G}^{\prime}{ }_{i}=\left.\mathbf{G}_{i}\right|_{\mathbf{P}=\mathbf{I}_{M}}$ is the nulling matrix in the case of $\mathbf{P}=\mathbf{I}_{M}$, and $(\cdot)_{i j}$ denotes the element of the matrix at the $i$ th row and $j$ th column. Except for determinations of $\mathbf{G}_{i}$ and $k_{i}$ described in (5) and (6), the detection algorithm for the MMSE case is the same as that for the ZF case in (2a)-(2k). For the MMSE case, the postdetection SINR $\rho_{k_{i}}$ for the $k_{i}$ th symbol is calculated as

$$
\rho_{k_{i}}=\frac{\left|\left(\mathbf{w}_{k_{i}}[\mathbf{H P}]_{\bar{k}_{i-1}}\right)_{1 k_{i}}\right|^{2}}{\sigma_{n}^{2}\left\|\mathbf{w}_{k_{i}}\right\|^{2}+\sum_{\ell \neq k_{i}}\left|\left(\mathbf{w}_{k_{i}}[\mathbf{H P}]_{\bar{k}_{i-1}}\right)_{1 \ell}\right|^{2}} .
$$


Note that the postdetection SINR $\rho_{k_{i}}$ depends on the transmit power for other symbols as well as that for the $k_{i}$ th symbol, in contrast to the ZF case.

\section{TRAnSMit POWER AllocAtion}

In Section III-A, we derive a TPA scheme that minimizes the BER. In the derivations, we assume that ZF is used for nulling, and the MMSE case is discussed in brief. In Section III-B, the results of Section III-A are applied to an uncoded quadrature amplitude modulation (QAM).

\section{A. Derivation of TPA Scheme}

To derive a TPA scheme, we first express the BER of each transmit symbol as a function of transmit power $\left\{P_{i}: i=\right.$ $1,2, \ldots, M\}$, and find $\left\{P_{i}\right\}$ that minimizes the overall BER. If the cancellations of previously detected symbols are perfect, the BER $E_{k_{i}}$ of the $k_{i}$ th transmit symbol for a given channel state may be represented as a function of the postdetection SINR in (4)

$$
E_{k_{i}}=f\left(\rho_{k_{i}}\right)=f\left(\frac{P_{k_{i}}}{\sigma_{n}^{2}\left\|\mathbf{v}_{k_{i}}\right\|^{2}}\right)
$$

where the function $f(\cdot)$ is determined by a specific modulation scheme. Taking the effects of error propagation into consideration, the actual BER $P_{b, k_{i}}(i=1,2, \ldots, M)$ of each detection stage for a given channel state may be approximated as

$$
\begin{aligned}
P_{b, k_{1}}= & E_{k_{1}} \\
P_{b, k_{2}}= & \left(1-P_{b, k_{1}}\right) E_{k_{2}}+P_{b, k_{1}} \alpha_{k_{2} \mid 1} \cong E_{k_{2}}+\alpha_{k_{2} \mid 1} P_{b, k_{1}} \\
P_{b, k_{3}}= & \left(1-P_{b, k_{1}}\right)\left(1-P_{b, k_{2}}\right) E_{k_{3}}+\left\{P_{b, k_{1}}\left(1-P_{b, k_{2}}\right)\right. \\
& \left.+\left(1-P_{b, k_{1}}\right) P_{b, k_{2}}\right\} \alpha_{k_{3} \mid 1}+P_{b, k_{1}} P_{b, k_{2}} \alpha_{k_{3} \mid 2} \\
\cong & E_{k_{3}}+\alpha_{k_{3} \mid 1}\left(P_{b, k_{1}}+P_{b, k_{2}}\right) \\
& \cdots \cdots \\
P_{b, k_{M}} \cong & E_{k_{M}}+\alpha_{k_{M} \mid 1}\left(P_{b, k_{1}}+P_{b, k_{2}}+\cdots+P_{b, k_{M-1}}\right)
\end{aligned}
$$

where $\alpha_{k_{i} \mid j}$ denotes the probability of bit error at the $i$ th detection stage, given that $j$ stages out of $(i-1)$ previous detection stages are erroneously detected. The approximations in (9) result from neglecting second and higher order terms of $E_{k_{i}}$ and $P_{b, k_{i}}$, which may be justified at high signal-to-noise ratio (SNR). Since the transmit symbols are independent of one another, the overall BER $P_{b}$ may be calculated as an arithmetic mean of the BER for every symbol

$$
\begin{aligned}
P_{b} & =\frac{1}{M} \sum_{i=1}^{M} P_{b, k_{i}} \\
& =\frac{1}{M} \sum_{i=1}^{M}\left(\prod_{j=1}^{M-i}\left(1+\alpha_{k_{M-j+1} \mid 1}\right)\right) \cdot f\left(\frac{P_{k_{i}}}{\sigma_{n}^{2}\left\|\mathbf{v}_{k_{i}}\right\|^{2}}\right) .
\end{aligned}
$$

We use the Lagrange multiplier method to find transmit power $\left\{P_{i}\right\}$ that minimizes the overall BER in (10) under the total transmit power constraint. The cost function may be expressed as

$$
J\left(P_{1}, P_{2}, \ldots, P_{M}\right)=P_{b}+\lambda\left(\sum_{i=1}^{M} P_{k_{i}}-M\right)
$$

where $\lambda$ is the Lagrange multiplier, and the total transmit power constraint is given as

$$
\sum_{i=1}^{M} P_{k_{i}}=M
$$

From $\partial J / \partial P_{k_{i}}=0$, a set of $M$ equations is found as

$$
\begin{aligned}
& \frac{d f}{d P_{k_{i}}}\left(\frac{P_{k_{i}}}{\sigma_{n}^{2}\left\|\mathbf{v}_{k_{i}}\right\|^{2}}\right)=-\frac{M \lambda}{\prod_{j=1}^{M-i}\left(1+\alpha_{k_{M-j+1} \mid 1}\right)}, \\
& i=1,2, \ldots, M \text {. }
\end{aligned}
$$

Solving $M+1$ simultaneous equations in (12) and (13), we can obtain a set of transmit power $\left\{P_{i}\right\}$ that minimizes the BER in (10).

As discussed in Section II, the postdetection SINR in (7) for the MMSE case depends on the transmit power for other symbols, as well as that for the corresponding symbol. In this case, the derivation of the TPA scheme is not tractable in a manner described above, and this necessitates an approximation of (7). Note that the postdetection SINR in (4) for the ZF case is expressed as a product of the transmit power of the corresponding symbol and the postdetection SINR obtained with equal TPA. This implies that the transmit power for each symbol affects the postdetection SINR as a scaling factor. We use this relationship to approximate (7), on the basis that the fundamental operation of MMSE is the same as that of ZF, except for the consideration of noise. Hence, the postdetection SINR $\rho_{k_{i}}$ in (7) is approximated as

$$
\rho_{k_{i}} \cong P_{k_{i}} \cdot \frac{\left|\left(\mathbf{v}_{k_{i}}[\mathbf{H}]_{\bar{k}_{i-1}}\right)_{1 k_{i}}\right|^{2}}{\sigma_{n}^{2}\left\|\mathbf{v}_{k_{i}}\right\|^{2}+\sum_{\ell \neq k_{i}}\left|\left(\mathbf{v}_{k_{i}}[\mathbf{H}]_{\bar{k}_{i-1}}\right)_{1 \ell}\right|^{2}}
$$

where $P_{k_{i}}$ is the transmit power, $\left|\left(\mathbf{v}_{k_{i}}[\mathbf{H}]_{\bar{k}_{i-1}}\right)_{1 k_{i}}\right|^{2}$ $/\left(\sigma_{n}^{2}\left\|\mathbf{v}_{k_{i}}\right\|^{2}+\sum_{\ell \neq k_{i}}\left|\left(\mathbf{v}_{k_{i}}[\mathbf{H}]_{\bar{k}_{i-1}}\right)_{1 \ell}\right|^{2}\right)$ is the postdetection SINR for $\mathbf{P}=\mathbf{I}_{M}$, and $\mathbf{v}_{k_{i}}$ is the weight vector for $\mathbf{P}=\mathbf{I}_{M}$. It should be noted that the approximation might not be justified at low SNR. With the approximation in (14), the transmit power can be calculated in a similar manner as the $\mathrm{ZF}$ case. Note that $\mathbf{v}_{k_{i}}$ and $\mathbf{w}_{k_{i}}$ should be separately calculated to determine the transmit power and to estimate the transmit symbols, respectively, since there is no explicit relationship between $\mathbf{v}_{k_{i}}$ and $\mathbf{w}_{k_{i}}$, in contrast to the $\mathrm{ZF}$ case. This may increase the computational requirements of the receiver compared with the case of the conventional V-BLAST system.

\section{B. Application to Uncoded QAM}

When uncoded $2^{R}$-ary QAM is employed for all symbols, the BER for the $k_{i}$ th symbol $x_{k_{i}}$ can be tightly approximated by an exponential function of $\rho_{k_{i}}$ as [11]

$$
E_{k_{i}} \cong \frac{1}{5} \exp \left(-\frac{1.6 \rho_{k_{i}}}{2^{R}-1}\right)=\frac{1}{5} \exp \left(-\frac{1.6 P_{k_{i}}}{\left(2^{R}-1\right) \Lambda_{k_{i}}}\right)
$$

and from (4) and (14), $\Lambda_{k_{i}}$ is defined as

$$
\Lambda_{k_{i}} \triangleq \begin{cases}\frac{\sigma_{n}^{2}\left\|\mathbf{v}_{k_{i}}\right\|^{2},}{\left(\sigma_{n}^{2}\left\|\mathbf{v}_{k_{i}}\right\|^{2}+\sum_{\ell \neq i}\left|\left(\mathbf{v}_{k_{i}}[\mathbf{H}]_{\bar{k}_{i-1}}\right)_{1 \ell}\right|^{2}\right)} & \text { ZF } \\ \left|\left(\mathbf{v}_{k_{i}}[\mathbf{H}]_{\bar{k}_{i-1}}\right)_{1 k_{i}}\right|^{2} & \text { MMSE . }\end{cases}
$$


In this case, (13) can be rewritten as

$$
\begin{aligned}
\frac{d}{d P_{k_{i}}} & \left(\frac{1}{5} \exp \left(-\frac{1.6 P_{k_{i}}}{\left(2^{R}-1\right) \Lambda_{k_{i}}}\right)\right) \\
& =-\frac{M \lambda}{\prod_{j=1}^{M-i}\left(1+\alpha_{k_{M-j+1} \mid 1}\right)}, \quad i=1,2, \ldots, M .
\end{aligned}
$$

By solving simultaneous equations in (12) and (17), we can find the solution for the transmit power as

$$
\begin{gathered}
P_{k_{i}}=-0.625\left(2^{R}-1\right) \Lambda_{k_{i}} \ln \left(\frac{3.125 M \lambda\left(2^{R}-1\right) \Lambda_{k_{i}}}{\prod_{j=1}^{M-i}\left(1+\alpha_{k_{M-j+1} \mid 1}\right)}\right), \\
i=1,2, \ldots, M \\
\lambda=\exp \left(-\frac{\frac{1.6 M}{\left(2^{R}-1\right)}+\sum_{i=1}^{M} \Lambda_{k_{i}} \ln \left(\frac{3.125 M\left(2^{R}-1\right) \Lambda_{k_{i}}}{\prod_{j=1}^{M-i}\left(1+\alpha_{k_{M-j+1} \mid 1}\right)}\right)}{\sum_{i=1}^{M} \Lambda_{k_{i}}}\right) .
\end{gathered}
$$

As shown in (16) and (18), $\Lambda_{k_{i}}(i=1,2, \ldots, M)$ are required in calculating $P_{k_{i}}$, and they are calculated using the nulling vectors $\mathbf{v}_{k_{i}}(i=1,2, \ldots, M)$. These nulling vectors can be calculated using the detection algorithm in (2a)-(2k) with $\mathbf{P}=\mathbf{I}_{M}$. Note that the conditional probabilities $\alpha_{k_{i} \mid 1}(i=2,3, \ldots, M)$ need to be predetermined to calculate the transmit power. If we set $\alpha_{k_{i} \mid 1}=0(i=2,3, \ldots, M)$ to ignore error propagations in the overall BER equation in (10), the TPA in (18) may be simplified to

$$
\begin{gathered}
P_{k_{i}}=-0.625\left(2^{R}-1\right) \Lambda_{k_{i}} \ln \left(3.125 M\left(2^{R}-1\right) \Lambda_{k_{i}}\right), \\
i=1,2, \ldots, M \\
\lambda=\exp \left(-\frac{\frac{1.6 M}{\left(2^{R}-1\right)}+\sum_{i=1}^{M} \Lambda_{k_{i}} \ln \left(3.125 M\left(2^{R}-1\right) \Lambda_{k_{i}}\right)}{\sum_{i=1}^{M} \Lambda_{k_{i}}}\right) .
\end{gathered}
$$

\section{NUMERICAL RESULTS}

In this section, the performance of the modified V-BLAST system with the proposed TPA is evaluated and compared with that of the conventional V-BLAST system with equal TPA. The performance of the maximum-likelihood (ML) detection with equal TPA is also presented for comparison purposes. Although the ML detection does not need feedback and provides the best performance among detection schemes of the conventional V-BLAST [12], it generally requires larger complexity than the V-BLAST detection. Quaternary phase-shift keying (QPSK) modulation $(R=2)$ is assumed to be employed, and the transmit power for the modified V-BLAST system is calculated using (19), unless explicitly specified. We use $(M, N)$ notation to represent a MIMO configuration with $M$ transmit and $N$ receive antennas.

Fig. 1 shows the effects of BER expressions for TPA on the overall uncoded BER performance of the modified V-BLAST for a $(4,4)$ MIMO system. For both ZF and MMSE nulling schemes, two BER curves are obtained through simulations using TPA in (18) and (19), respectively. It is observed that the two BER curves are almost indistinguishable for both the ZF

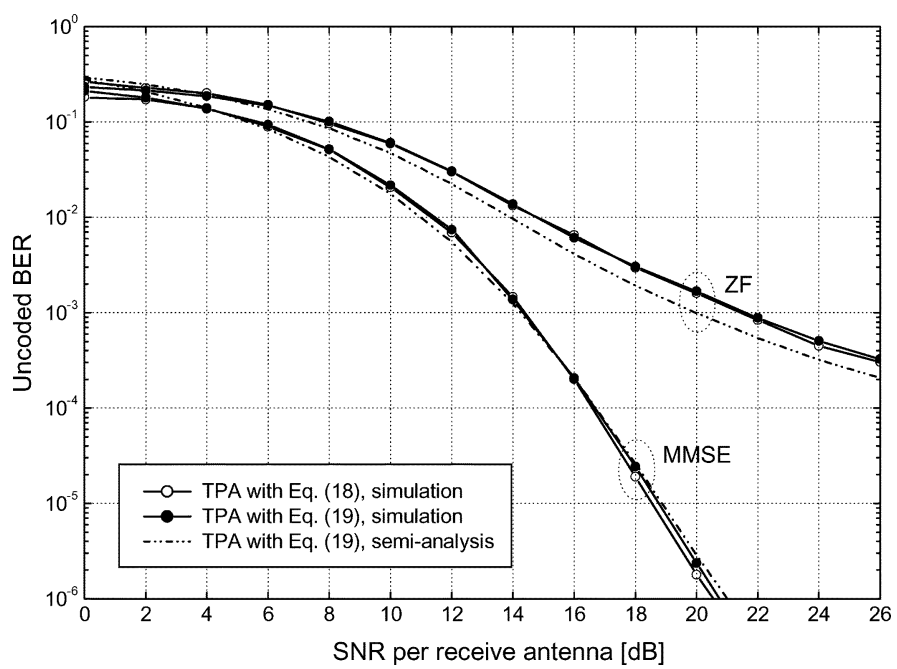

Fig. 1. Effects of BER expressions for TPA on the uncoded BER performance of the modified V-BLAST for a $(4,4)$ MIMO system.

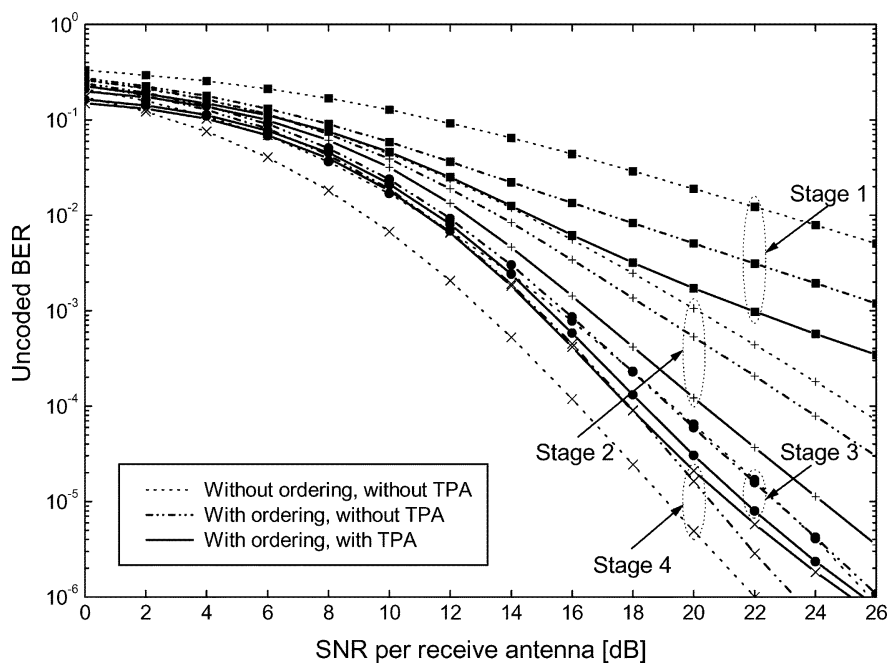

Fig. 2. Effects of detection ordering and proposed TPA on the uncoded BER performance of each detection stage for a $(4,4)$ MIMO system with ZF nulling, when error propagation is ignored.

and MMSE cases. This indicates that it hardly affects the performance of TPA, whether the error propagation is considered or not in the BER equation. Based on this observation, we use the simpler (19) rather than (18) in the subsequent results. In Fig. 1, we also verify that the semianalytic results agree well with the simulation results, especially for the MMSE case. A slight difference may be due to the approximations in (9) and Gaussian approximation of interference in (15).

Fig. 2 shows the effects of the detection ordering and proposed TPA on the uncoded BER performance of each detection stage for a $(4,4)$ MIMO system with ZF nulling. Note that only the detection ordering is used for the conventional V-BLAST system, whereas both the detection ordering and proposed TPA are used for the modified V-BLAST system. Error propagation is ignored in calculating the BER for each stage, and the results are obtained through semianalytic methods using (15) and (16). Without the detection ordering and TPA, the BERs for earlier detection stages are shown to decrease more slowly with SNR per receive antenna $\left(M / \sigma_{n}^{2}\right)$ than the BERs for later stages. This verifies that the earlier detection stage has the lower diversity 


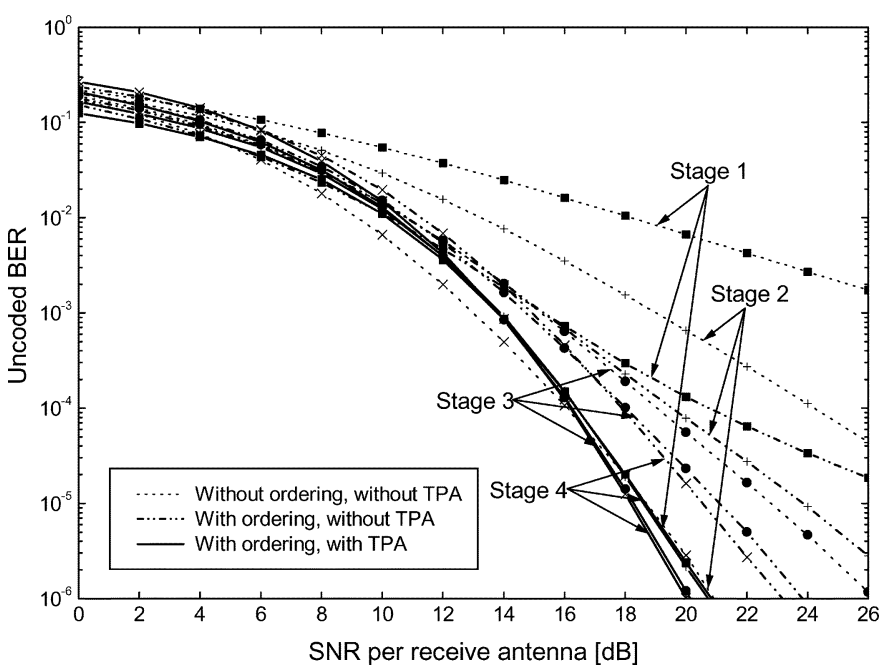

Fig. 3. Effects of detection ordering and proposed TPA on the uncoded BER performance of each detection stage for a $(4,4)$ MIMO system with MMSE nulling, when error propagation is ignored.

order. The use of detection ordering is found to decrease the BER of the first and second detection stages, and increases that of the fourth stage. It is interesting to note that the detection ordering does not change the diversity order, but increases the average SINR for early detection stages, since the detection ordering just shifts the BER curves without changing the slope. When the proposed TPA is incorporated, further improvement for the first and second stages is observed. The major impact of the proposed TPA scheme is also shown to increase the average SINR for early detection stages. Since the overall BER performance is limited by early detection stages, the proposed TPA scheme is expected to decrease the overall BER. Furthermore, more reliable decisions in early detection stages may reduce the effects of error propagation.

Fig. 3 shows the effects of the detection ordering and proposed TPA for the MMSE case under the same conditions as in Fig. 2. In the absence of TPA, the effect of detection ordering is shown to be similar to the ZF case. However, the improvement in low detection stages with the MMSE nulling is shown to be much more significant than with the ZF nulling. When both the detection ordering and proposed TPA schemes are employed, we can observe further significant improvement for all the detection stages. It is remarkable that the performance of all the detection stages is very similar to one another, and it is better than that of the last detection stage without TPA at high SNR region, unlike the ZF case. The reason for this is that disparities in the interference-plus-noise power among detection stages, $\Lambda_{k_{i}}$ 's in (16), are much smaller with the MMSE than with the ZF nulling, due to reduced noise enhancement. The smaller disparities in $\Lambda_{k_{i}}$ 's make the TPA the more effective in compensating for the worst detection stage, so that it no longer limits the overall performance. In Fig. 3, it should also be noted that the last detection stage without TPA achieves the same diversity order of $N$ as the ML detection scheme. This implies that the TPA with the MMSE nulling can realize a greater diversity order than the ML detection. ${ }^{1}$ This is because the TPA reduces deep fades in the received signal, increasing the diversity order.

\footnotetext{
${ }^{1}$ This diversity advantage over the ML detection may diminish and even disappear if the input is space-time coded.
}

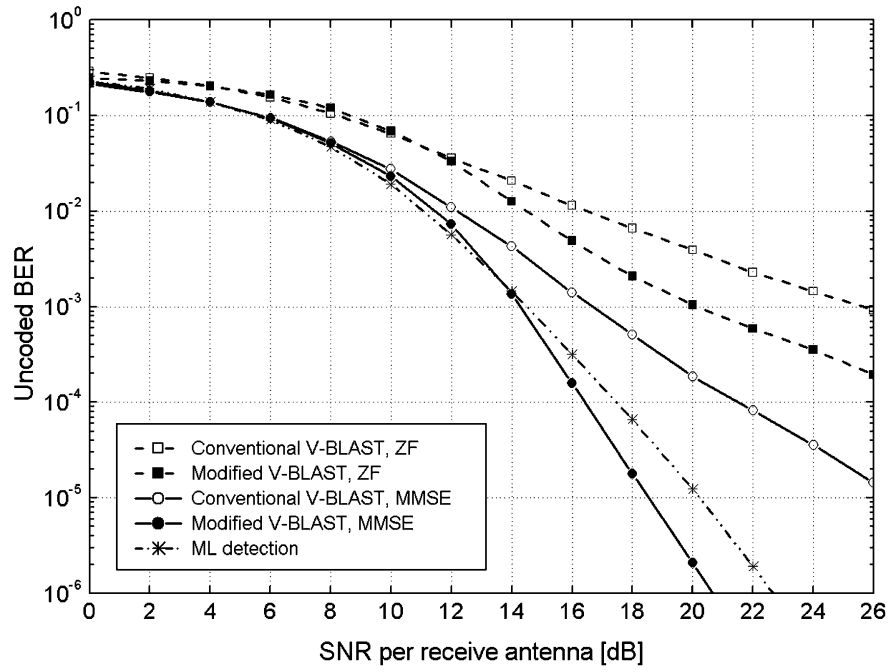

Fig. 4. Uncoded BER performance for a $(4,4)$ MIMO system.

The effect of TPA on diversity order is more remarkable for the MMSE than for the ZF, due to smaller disparities in $\Lambda_{k_{i}}$ 's in (16). Hence, the modified V-BLAST system with the MMSE nulling achieves a form of diversity due to TPA as well as inherent receive diversity, realizing a greater diversity order than the ML detection that achieves only receive diversity.

In Fig. 4, the overall uncoded BER performance of the $(4,4)$ modified V-BLAST system with the proposed TPA scheme is compared with that of the conventional V-BLAST system. The effects of error propagation are not neglected, and simulations are used to obtain the actual performance. Comparing Fig. 4 with Figs. 2 and 3, it can be seen that the overall BER is mainly determined from the BER for the first detection stage. The modified V-BLAST system is shown to significantly outperform the conventional one. When the ZF nulling is used, the SNR gain is about $4.0 \mathrm{~dB}$ at BER of $10^{-3}$. In the case of the MMSE, the modified V-BLAST system provides $2.5 \mathrm{~dB}$ SNR gain at BER of $10^{-3}$. Furthermore, the modified V-BLAST system with the MMSE nulling is shown to provide almost the same performance as the ML detection at low SNR values, and outperform the ML detection for the SNR greater than $14 \mathrm{~dB}$. This is because the modified V-BLAST system with the MMSE nulling realizes a greater diversity order than the ML detection, as explained previously.

\section{CONCLUSIONS}

In this letter, we have presented a modification of V-BLAST, and proposed an effective TPA scheme that minimizes the BER averaged over all detection stages. The feedback overhead for the proposed TPA scheme is relatively small. It has been found that the TPA scheme in combination with detection ordering improves the performance of lower detection stages, resulting in significant reduction in the overall uncoded BER. Simulation results have shown that the modified V-BLAST system with the proposed TPA scheme achieves $2.5-4.0 \mathrm{~dB}$ of SNR gain over the conventional V-BLAST system at uncoded BER of $10^{-3}$. With the MMSE nulling, the modified V-BLAST system has been found to achieve a diversity gain as well as the average SNR gain, resulting in performance comparable to or better than that of complex ML detection, which does not require any feedback. 
Finally, it should be pointed out that the above results have been derived under ideal conditions. An important topic for future work is to investigate the impacts of practical conditions, such as imperfect channel estimation, feedback delay, quantization errors, and spatial correlation of MIMO channel.

\section{ACKNOWLEDGMENT}

The authors would like to thank the Editor and anonymous reviewers for their helpful comments.

\section{REFERENCES}

[1] G. J. Foschini and M. J. Gans, "On limits of wireless communications in a fading environment when using multiple antennas," Wireless Pers. Commun., vol. 6, pp. 315-335, Mar. 1998.

[2] V. Tarokh, A. Naguib, and N. Seshadri, "Combined array processing and space-time coding," IEEE Trans. Inform. Theory, vol. 45, pp. 1121-1128, May 1999.

[3] G. G. Raleigh and J. M. Cioffi, "Spatio-temporal coding for wireless communication," IEEE Trans. Commun., vol. 46, pp. 357-366, Mar. 1998.

[4] G. J. Foschini, "Layered space-time architecture for wireless communication in a fading environment when using multiple antennas," Bell Labs Tech. J., vol. 1, pp. 41-59, 1996.
[5] P. W. Wolniansky, G. J. Foschini, G. D. Golden, and R. A. Valenzuela, "V-BLAST: An architecture for realizing very high data rates over the rich-scattering wireless channel," in Proc. URSI Int. Symp. Signals, Systems, Electronics, Pisa, Italy, Sept.-Oct. 1998, pp. 295-300.

[6] M. F. Demirkol and M. A. Ingram, "Power-controlled capacity for interfering MIMO links," in Proc. IEEE Vehicular Technology Conf., Atlantic City, NJ, Oct. 2001, pp. 187-191.

[7] S. T. Chung, A. Lozano, and H. C. Howard, "Approaching eigenmode BLAST channel capacity using V-BLAST with rate and power feedback," in Proc. IEEE Vehicular Technology Conf., Atlantic City, NJ, Oct. 2001, pp. 915-919.

[8] K. J. Hwang and K. B. Lee, "Transmit power allocation with small feedback overhead for a multiple antenna system," in Proc. IEEE Vehicular Technology Conf., Vancouver, BC, Canada, Sept. 2002, pp. 2158-2162.

[9] W.-J. Choi, R. Negi, and J. M. Cioffi, "Combined ML and DFE decoding for the V-BLAST system," in Proc. IEEE Int. Conf. Communications, New Orleans, LA, June 2000, pp. 1243-1248.

[10] S. Catreux, P. F. Driessen, and L. J. Greenstein, "Data throughputs using multiple-input multiple-output (MIMO) techniques in a noise-limited cellular environment," IEEE Trans. Wireless Commun., vol. 1, pp. 226-235, Apr. 2002.

[11] S. T. Chung and A. J. Goldsmith, "Degree of freedom in adaptive modulation: A unified view," IEEE Trans. Commun., vol. 49, pp. 1561-1571, Sept. 2001.

[12] X. Zhu and R. D. Murch, "Multi-input multi-output maximum-likelihood detection for a wireless system," in Proc. IEEE Vehicular Technology Conf., Rhodes, Greece, May 2001, pp. 137-141. 